\title{
The Effect of Extracorporeal Shock Wave Therapy on Lower Limb Spasticity in Subacute Stroke Patients
}

\author{
Seung Won Moon, $\mathrm{MD}^{1}$, Jin Hoan Kim, $\mathrm{MD}^{1}$, Mi Jin Jung, $\mathrm{MD}^{1}$, Seungnam Son, $\mathrm{MD}^{2}$, Joong Hoon Lee, $\mathrm{MD}^{3}$, \\ Heesuk Shin, $\mathrm{MD}^{1}$, Eun Shin Lee, $\mathrm{MD}^{1}$, Chul Ho Yoon, $\mathrm{MD}^{1}$, Min-Kyun Oh, $\mathrm{MD}^{1}$ \\ ${ }^{1}$ Department of Rehabilitation Medicine and Institute of Health Sciences, Gyeongsang National University Graduate School of \\ Medicine, ${ }^{2}$ Department of Neurology, Gyeongsang National University Graduate School of Medicine, Jinju, \\ ${ }^{3}$ Department of Rehabilitation Medicine, Samcheonpo Seoul Hospital, Seoul, Korea
}

Objective To evaluate the effect of extracorporeal shock wave therapy (ESWT) on lower limb spasticity in subacute stroke patients.

Methods We studied thirty hemiplegic subacute stroke patients with ankle plantar flexor spasticity. ESWT was applied for 1 session/week, with a total of 3 sessions at the musculotendinous junction of medial and lateral gastrocnemius muscles. Patients were evaluated both clinically and biomechanically at baseline, after sham stimulation, and at immediately 1 week and 4 weeks after ESWT. For clinical assessment, Modified Ashworth Scale (MAS), clonus score, passive range of motion of ankle, and Fugl-Myer Assessment for the lower extremity were used. A biomechanical assessment of spasticity was conducted by an isokinetic dynamometer. Two parameters, peak eccentric torque (PET) and torque threshold angle (TTA), were analyzed at the velocities of $60^{\circ} / \mathrm{sec}, 180^{\circ} / \mathrm{sec}$, and $240^{\circ} / \mathrm{sec}$.

Results After sham stimulation, there were no significant changes between each assessment. MAS and PET $\left(180^{\circ} /\right.$ sec and $240^{\circ}$ /sec) were significantly improved immediately and 1 week after ESWT. However, these changes were not significant at 4 weeks after ESWT. PET $\left(60^{\circ} / \mathrm{sec}\right)$ and TTA $\left(60^{\circ} / \mathrm{sec}, 180^{\circ} / \mathrm{sec}\right.$, and $\left.240^{\circ} / \mathrm{sec}\right)$ were significantly improved immediately after ESWT. Yet, these changes were not significant at 1 week and 4 weeks after ESWT as well. Conclusion Lower limb spasticity in subacute stroke patients was significantly improved immediately after ESWT. Although the therapeutic effect of ESWT reduced with time and therefore was not significant at 4 weeks after ESWT, the degree of spasticity was lower than that of the baseline. Future studies with a larger sample of patients are warranted in order to verify the protocols which can optimize the effect of ESWT on spasticity.

Keywords High-energy shock waves, Muscle spasticity, Stroke

Received January 31, 2013; Accepted May 14, 2013

Corresponding author: Min-Kyun Oh

Department of Rehabilitation Medicine and Institute of Health Sciences, Gyeongsang National University Graduate School of Medicine, 15 Jinju-daero 816beon-gil, Jinju 660-702, Korea

Tel: +82-55-750-8854, Fax: +82-55-750-8255, E-mail: solioh21@naver.com

(c) This is an open-access article distributed under the terms of the Creative Commons Attribution Non-Commercial License (http://creativecommons.org/ licenses/by-nc/3.0) which permits unrestricted noncommercial use, distribution, and reproduction in any medium, provided the original work is properly cited. Copyright $\odot 2013$ by Korean Academy of Rehabilitation Medicine 


\section{INTRODUCTION}

Spasticity, which is a component of the upper motor neuron syndrome, is a neurological symptom frequently appearing in stroke patients and is defined as a velocity dependent increase in muscle tone with exaggerated tendon reflexes [1]. It has been reported that spasticity appears in approximately $35 \%$ of stroke patients. Spasticity should be appropriately treated because it inhibits normal movements and causes pain or postural abnormality, thereby hindering patients' ability for daily living and affecting the quality of life of patients [2]. The existing controlling and treatment methods for spasticity include physical therapy, oral anti-spasticity drugs, such as dantrolene, benzodiazepine, baclofen, tizanidine, and clonidine, chemical nerve block and motor point block using phenol or ethyl alcohol, and botulinum toxin injection [3]. The necessity of new noninvasive treatment methods for spasticity has been raised because spasticity cannot be controlled sometimes even with diverse treatment methods as well as due to the existing side effects of oral drugs and the invasiveness of local treatment methods.

After being proved to be effective on the delayed union of fractures [4], extracorporeal shock wave therapy (ESWT) has been used in diverse musculoskeletal diseases, such as plantar fasciitis, lateral epicondylitis, and calcific tendinitis [5-7]. As recent studies indicate that ESWT is also effective in treating spasticity, ESWT is being considered as a new treatment method for spasticity. A total of four studies have applied ESWT to stroke patients with spasticity thus far. Of them, three studies applied ESWT to the treatment of upper limb spasticity and one applied it to the treatment of lower limb spasticity [811]. Only clinical assessments have been used to determine the effects of spasticity treatment in all studies, and no biomechanical assessment has been conducted in any of the studies. A study on lower limb spasticity evaluated only the effects immediately after treatment and did not check whether the treatment effects were maintained thereafter. Therefore, the present study was intended to evaluate the effect of ESWT on lower limb spasticity in subacute stroke patients by conducting both clinical and biomechanical evaluations to check the treatment effects as well as to observe whether the effects are maintained.

\section{MATERIALS AND METHODS}

\section{Subjects}

The subjects of the present study included 30 subacute stroke patients ( 17 males and 13 females, mean age of $52.6 \pm 14.9$ years) with spasticity in the ankle plantar flexor. Of the subjects, 16 patients had ischemic stroke and 14 patients had hemorrhagic stroke. Their average duration of illness after stroke was $80.5 \pm 46.5$ days (Table 1 ). The inclusion criteria in the study were as follows: patients aged at least 18 years, but not older than 80 years, who could be treated with shock waves and had agreed to the treatment, had a stroke at least one month prior to the study, and showed a Modified Ashworth Scale (MAS) score of the ankle exceeding 1+. Patients with ankle contracture or severe inflammation in treatment sites, and those with a history of botulinum toxin, phenol, or alcohol injections were excluded from the study. During the treatment period, range of motion (ROM) exercises, stretching, and physical therapy were equally implemented and the doses of medication which might affect spasticity were not changed.

The purpose and process of the study were sufficiently explained to all subject patients and the study was conducted with those who agreed to the study.

\section{Method}

\section{ESWT protocol}

In the ESWT, a PiezoWave (Richard Wolf GmbH, Knittlingen, Germany) was used to provide stimulation. The musculotendinous junction of the triceps surae muscle was identified using Accuvix XQ (Medison, Seoul, Korea), which is a diagnostic ultrasonography for the musculoskeletal system, and then marked. The musculotendinous junctions of the medial gastrocnemius and the lateral

Table 1. General characteristics of the subjects

\begin{tabular}{lc}
\hline \multicolumn{1}{c}{ Characteristic } & Value \\
\hline Age (yr) & $52.6 \pm 14.9$ \\
Sex (M:F) & $17: 13$ \\
Mean duration after stroke onset (day) & $80.5 \pm 46.5$ \\
Stroke subtype & \\
$\quad$ Infarction & 16 \\
Hemorrhage & 14 \\
Lesion site (right:left) & $12: 18$ \\
\hline Values are presented as mean+standard deviation.
\end{tabular}

Values are presented as mean \pm standard deviation. 
gastrocnemius were stimulated at $4 \mathrm{~Hz}, 1,500$ times with the intensity of stimulation using energy of $0.089 \mathrm{~mJ} / \mathrm{mm}^{2}$ within a range causing tolerable pain. Sham stimulation was provided by only making sound without getting the device into contact with the skin at the stimulation site.

According to the treatment protocol, sham stimulation was conducted at the first week, and three subsequent ESWT sessions were followed, one session per week. This protocol allows to secure the uniformity of the experimental and the control group as well as to determine the accurate treatment effects.

\section{Clinical and biomechanical assessment}

The treatment effects were evaluated five times in total; the clinical and biomechanical assessment of spasticity were conducted before the sham stimulation, immediately after the sham stimulation, immediately after the completion of the ESWT sessions, one week after the completion of the ESWT sessions, and four weeks after the completion of the ESWT sessions (Fig. 1).

The MAS, clonus scores (CS), the passive ROM of the ankle, and Fugl-Myer Assessment (FMA) of the lower limb were used for the clinical evaluation. For convenience in the statistical analysis, MAS $1+$ was substituted by 2 , and 2,3 , and 4 were substituted by 3,4 , and 5 , respectively. CS was given based on whether the ankle clonus appeared when a passive joint motion had been made as quickly as possible from the maximum plantarflexion to the maximum dorsiflexion while the patient was relaxed; 0 point was given to cases where there was no ankle clonus, 1 point when the ankle clonus was not maintained, 2 points when the ankle clonus was maintained, and 3 points when the ankle clonus appeared even after a slight contact or when it spontaneously appeared. The passive ROM of the ankle were obtained by measuring the angles of the maximum plantarflexion and the maximum dorsiflexion using a goniometer and summing up the angles. FMA is an indicator for the evaluation of the degree of motor function recovery after stroke, consisting of 33 upper limb performance items and 17 lower limb performance items, which intended to evaluate the quality of movements, reflex, and coordination. Each item is scored using a 3 point scale $(0-2)$. The maximum score of the upper limb is 66 points and that of the lower limb is 34 points. In the present study, only the lower limb items were used for evaluation [12].

In the biomechanical assessment, the spasticity of the affected side ankle plantar flexor muscle was quantitatively measured using a Biodex system 4 (Biodex Medical

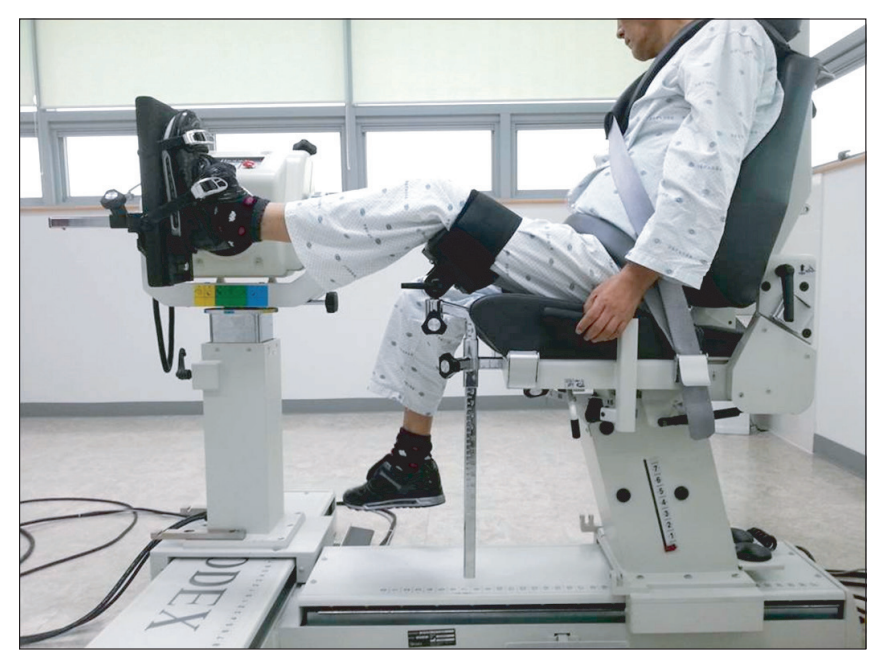

Fig. 2. Patient's posture of biomechanical assessment using the Biodex system isokinetic dynamometer.

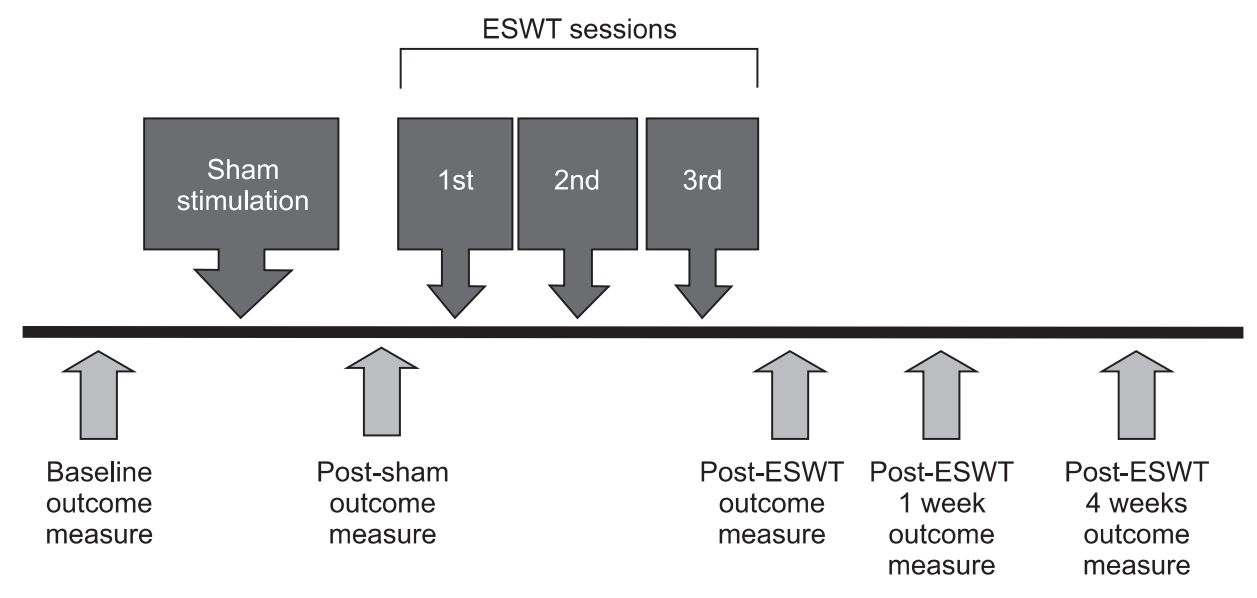

Fig. 1. Outline of treatment session with data collection. All patients were evaluated at baseline, after sham stimulation, and at immediately 1 week and 4 weeks after extracorporeal shock wave therapy (ESWT). 
System, Shirley, NY, USA) isokinetic dynamometer. Each subject patient was instructed to sit on an isokinetic dynamometer chair, and his/her trunk was erected upright and fixed to the back of the chair using a 3-point belt. The examiner fixed the knee joint of the lower limb of the subject to a $30^{\circ}$ flexion using Velcro tape, aligned the axis of the ankle joint with the axis of the isokinetic dynamometer, and fixed the foot to the connection terminal for ankle joints of the isokinetic dynamometer. The subjects were placed on the examination table of the isokinetic dynamometer in the same posture and were sufficiently stabilized. Then, tests were conducted while the ankle plantar flexor muscle's voluntary contraction was being eliminated (Fig. 2). In the tests, isokinetic passive joint movements from $30^{\circ}$ plantarflexion to $20^{\circ}$ dorsiflexion were made with the ankle joint of the lower limb being tested at angular speeds of $60^{\circ}, 180^{\circ}$, and $240^{\circ}$ per second to stretch the ankle plantar flexor muscle while measuring the eccentric torque values at every $0.5^{\circ}$ change of the joint angle. The joint angle was measured using $30^{\circ}$ plantarflexion of the ankle joint as a reference point $\left(0^{\circ}\right)$. The tests were conducted five times repeatedly at each angular speed; the averages of the three tests, excluding the first results and the last results, were obtained. At this time, the gravity of the connection terminal between the foot and the isokinetic dynamometer was compensated. To evaluate the spasticity, peak eccentric torque (PET) values and torque threshold angles (TTAs) were measured as parameters. The PET is the peak value of the eccentric torque occurring during passive joint movements. It is an indicator that presents the resistance to passive joint movements or muscle stretches, which is one of the major clinical symptoms of spasticity as quantified values. The TTA is the joint angle at points where the eccentric torque begins to occur. This value is an indicator that indirectly shows decreases in the threshold of the stretch reflex of the ankle plantar flexor muscle that shows spasticity, or the exaggeration of the stretch reflex. As the spasticity becomes more severe, the PET values increase and the TTAs decrease [13]. To eliminate interrater differences, all the evaluations were conducted by one doctor of the department of rehabilitation medicine.

\section{Statistical processing}

Paired t-tests were conducted for the statistical analysis using SPSS ver. 17.0 (SPSS Inc., Chicago, IL, USA), and the statistical significance level was set to $\mathrm{p}<0.05$.

\section{RESULTS}

\section{Clinical assessment}

Modified Ashworth scale of the ankle joint flexor muscle

MAS scores obtained in the basal evaluation before treatment, and evaluations after the sham stimulation, immediately after the treatment, one week after the treatment, and four weeks after the treatment were $2.50 \pm 0.67$, $2.50 \pm 0.67,1.41 \pm 0.67,1.67 \pm 0.65$, and $1.75 \pm 0.62$, respectively. Compared to the basal evaluation, the MAS scores statistically significantly decreased immediately after the shock wave treatment $(\mathrm{p}=0.002)$ and one week after the treatment $(\mathrm{p}=0.02)$. The MAS scores also decreased at four weeks after the treatment compared to the basal evaluation; however, the differences were not statistically significant (Fig. 3).

\section{Clonus score}

CSs obtained in the basal evaluation before treatment, and evaluations after the sham stimulation, immediately after the treatment, one week after the treatment, and four weeks after the treatment were $1.42 \pm 0.67,1.42 \pm 0.67$, $1.08 \pm 0.51,1.17 \pm 0.58$, and $1.17 \pm 0.58$, respectively. Compared to the basal evaluation, the values showed a decreasing tendency after the shock wave treatment although the differences were not significant.

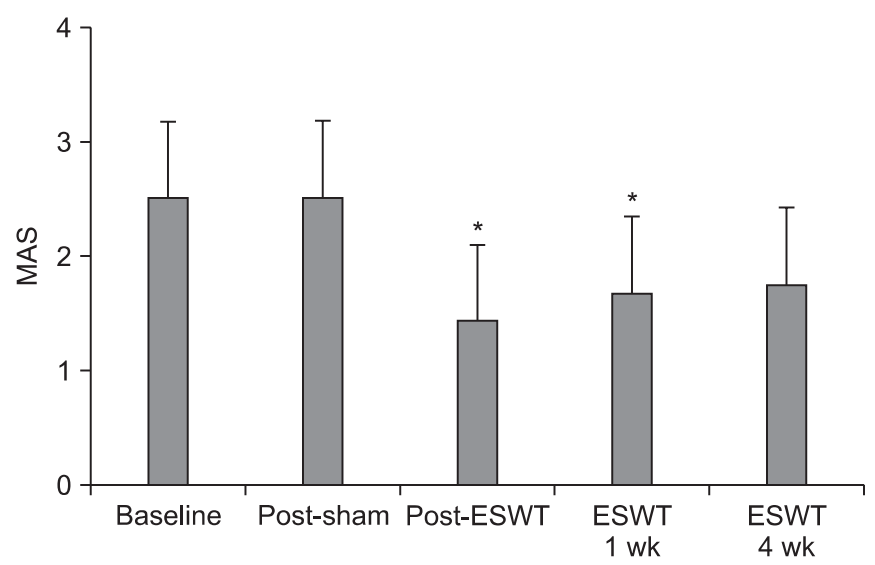

Fig. 3. Modified Ashworth Scale (MAS) of ankle plantar flexor before and after treatment. MAS was significantly improved immediately and 1 week after extracorporeal shock wave therapy (ESWT), but was not significantly changed at 4 weeks after ESWT. *Statistically significant compared with baseline $(\mathrm{p}<0.05)$. 


\section{Passive range of motion of the ankle}

PROM values obtained in the basal evaluation before treatment, and evaluations after the sham stimulation, immediately after the treatment, one week after the treatment, and four weeks after the treatment were $56.67 \pm 15.42^{\circ}, 56.67 \pm 15.42^{\circ}, 59.16 \pm 14.59^{\circ}, 59.58 \pm 13.56^{\circ}$, and $60.41 \pm 13.56^{\circ}$, respectively. Compared to the basal evaluation, the values showed an increasing tendency after the shock wave treatment although the differences were not significant.

\section{Fugl-Myer assessment of the lower limb}

FMA values obtained in the basal evaluation before treatment, and evaluations after the sham stimulation, immediately after the treatment, one week after the treatment, and four weeks after the treatment were $17.58 \pm 4.64,17.50 \pm 4.71,19.25 \pm 4.52,20.58 \pm 4.32$, and $20.92 \pm 4.34$, respectively. Compared to the basal evaluation, the values showed an increasing tendency after the shock wave treatment although the differences were not significant (Table 2).

\section{Biomechanical assessment}

\section{Peak eccentric torque}

At the angular speed $60^{\circ} / \mathrm{sec}$, the PET values obtained in the basal evaluation before treatment, and evaluations after the sham stimulation, immediately after the treatment, one week after the treatment, and four weeks after the treatment were $7.89 \pm 1.70,7.90 \pm 1.64,5.42 \pm 1.54$, $6.13 \pm 1.57$, and $6.44 \pm 1.68 \mathrm{Nm}$, respectively. At the angular speed $180^{\circ} / \mathrm{sec}$, the values were $8.62 \pm 1.53,8.68 \pm 1.57$, $6.38 \pm 1.61,6.87 \pm 1.56$, and $7.31 \pm 1.58 \mathrm{Nm}$, respectively. At the angular speed $240^{\circ} / \mathrm{sec}$, the values were $9.08 \pm 1.65$, $8.95 \pm 1.52,6.73 \pm 1.49,7.28 \pm 1.39$, and $7.74 \pm 1.50 \mathrm{Nm}$, respectively. At the angular speed $60^{\circ} / \mathrm{sec}$, compared to the basal evaluation, the PET values statistically significantly decreased only immediately after the treatment $(\mathrm{p}=0.004)$. At the angular speed $180^{\circ} / \mathrm{sec}$, compared to the basal evaluation, the PET values statistically significantly decreased immediately after the treatment $(\mathrm{p}=0.008)$ and at one week after the treatment $(\mathrm{p}=0.03)$. At the angular speed $240^{\circ} / \mathrm{sec}$, compared to the basal evaluation, the PET values statistically significantly decreased immediately after the treatment $(\mathrm{p}=0.008)$ and at one week after the treatment $(\mathrm{p}=0.03)$. At four weeks after the treatment, although the values decreased compared to the basal evaluation, the differences were not statistically significant (Fig. 4).

\section{Torque threshold angle}

At the angular speed $60^{\circ} / \mathrm{sec}$, the TTAs obtained in the basal evaluation before treatment, and evaluations after the sham stimulation, immediately after the treatment, one week after the treatment, and four weeks after the

Table 2. Value of clinical and biomechanical assessments

\begin{tabular}{|lccccc}
\hline & Baseline & Post-sham & Post-ESWT & Post 1 wk & Post 4 wk \\
\hline MAS & $2.50 \pm 0.67$ & $2.50 \pm 0.67$ & $1.41 \pm 0.67^{\text {a) }}$ & $1.67 \pm 0.65^{\text {a) }}$ & $1.75 \pm 0.62$ \\
\hline Clonus score & $1.42 \pm 0.67$ & $1.42 \pm 0.67$ & $1.08 \pm 0.51$ & $1.17 \pm 0.58$ & $1.17 \pm 0.58$ \\
\hline PROM $\left({ }^{\circ}\right)$ & $56.67 \pm 15.42$ & $56.67 \pm 15.42$ & $59.16 \pm 14.59$ & $59.58 \pm 13.56$ & $60.41 \pm 13.56$ \\
\hline FMA & $17.58 \pm 4.64$ & $17.50 \pm 4.71$ & $19.25 \pm 4.52$ & $20.58 \pm 4.32$ & $20.92 \pm 4.34$ \\
\hline PET $(\mathrm{Nm})$ & & & & \\
\hline 60 & $7.89 \pm 1.70$ & $7.90 \pm 1.64$ & $5.42 \pm 1.54^{\text {a) }}$ & $6.13 \pm 1.57$ & $6.44 \pm 1.68$ \\
\hline 180 & $8.62 \pm 1.53$ & $8.68 \pm 1.57$ & $6.38 \pm 1.61^{\text {a) }}$ & $6.87 \pm 1.56^{\text {a) }}$ & $7.31 \pm 1.58$ \\
\hline 240 & $9.08 \pm 1.65$ & $8.95 \pm 1.52$ & $6.73 \pm 1.49^{\text {a) }}$ & $7.28 \pm 1.39^{\text {a) }}$ & $7.74 \pm 1.50$ \\
\hline TTA $\left({ }^{\circ}\right)$ & & & & \\
\hline 60 & $12.08 \pm 3.48$ & $12.33 \pm 3.52$ & $16.58 \pm 2.84^{\text {a) }}$ & $15.42 \pm 2.87$ & $14.75 \pm 2.90$ \\
\hline 180 & $9.33 \pm 2.83$ & $8.33 \pm 3.42$ & $12.92 \pm 3.18^{\text {a) }}$ & $11.67 \pm 2.46$ & $10.67 \pm 2.10$ \\
\hline 240 & $7.25 \pm 2.18$ & $7.25 \pm 2.45$ & $12.00 \pm 3.19^{\text {a) }}$ & $9.83 \pm 2.66$ & $9.17 \pm 1.95$ \\
\hline
\end{tabular}

Values are presented as mean \pm standard deviation.

ESWT, extracorporeal shock wave therapy; MAS, Modified Ashworth Scale; PROM, passive range of motion of ankle; FMA, Fugl-Myer assessment of the lower extremity; PET, peak eccentric torque; TTA, torque threshold angle.

${ }^{a)}$ Statistically significant compared with baseline $(\mathrm{p}<0.05)$. 


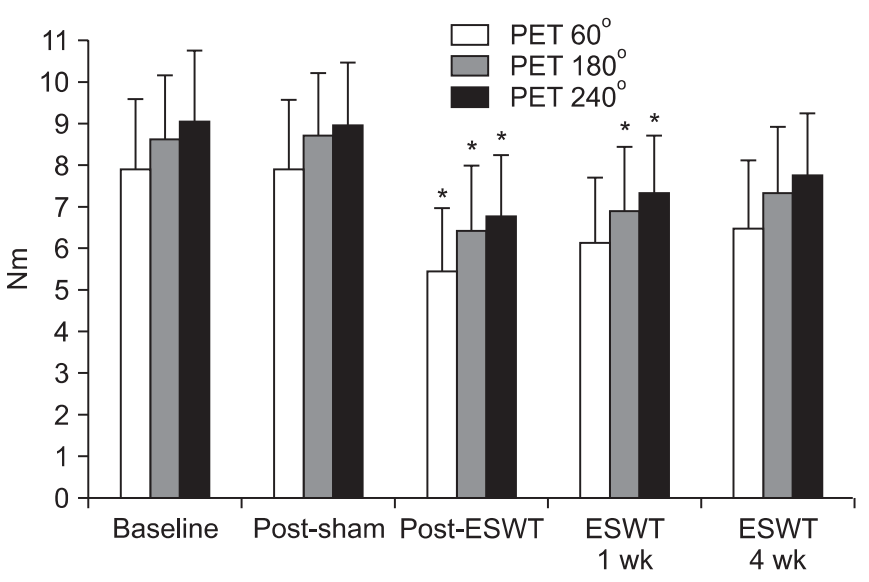

Fig. 4. Peak eccentric torque (PET) of ankle plantar flexor before extracorporeal shock wave therapy (ESWT) and after sham stimulation, and at immediately 1 week and 4 weeks after ESWT. *Statistically significant compared with baseline $(\mathrm{p}<0.05)$.

treatment were $12.08 \pm 3.48^{\circ}, 12.33 \pm 3.52^{\circ}, 16.58 \pm 2.84^{\circ}$, $15.42 \pm 2.87^{\circ}$, and $14.75 \pm 2.90^{\circ}$, respectively. At the angular speed $180^{\circ} / \mathrm{sec}$, the values were $9.33 \pm 2.83^{\circ}, 8.33 \pm 3.42^{\circ}$, $12.92 \pm 3.18^{\circ}, 11.67 \pm 2.46^{\circ}$, and $10.67 \pm 2.10^{\circ}$, respectively. At the angular speed $240^{\circ} / \mathrm{sec}$, the values were $7.25 \pm 2.18^{\circ}$, $7.25 \pm 2.45^{\circ}, 12.00 \pm 3.19^{\circ}, 9.83 \pm 2.66^{\circ}$, and $9.17 \pm 1.95^{\circ}$, respectively. At the angular speed $60^{\circ} / \mathrm{sec}, 180^{\circ} / \mathrm{sec}$, and $240^{\circ} / \mathrm{sec}$, compared to the basal evaluation, the TTAs increased statistically significantly only immediately after the treatment. At the angular speeds $60^{\circ} / \mathrm{sec}, 180^{\circ} / \mathrm{sec}$, and $240^{\circ} / \mathrm{sec}$, the p-values were $0.008,0.020$, and 0.001 , respectively. At one week and four weeks after the treatment, although the values increased compared to the basal evaluation, the differences were not statistically significant (Fig. 5).

\section{DISCUSSION}

The present study was aimed to evaluate the effect of ESWT on lower limb spasticity in subacute stroke patients and to conduct both clinical and biomechanical evaluations in order to check the treatment effects and its maintenance. The musculotendinous junction of the medial gastrocnemius and the lateral gastrocnemius were stimulated with $4 \mathrm{~Hz}, 1,500$ times, and $0.089 \mathrm{~mJ} / \mathrm{mm}^{2}$ once each week for three weeks in total.

The results conveyed that lower limb spasticity significantly improved immediately after the ESWT treatment in subacute stroke patients. However, its therapeutic

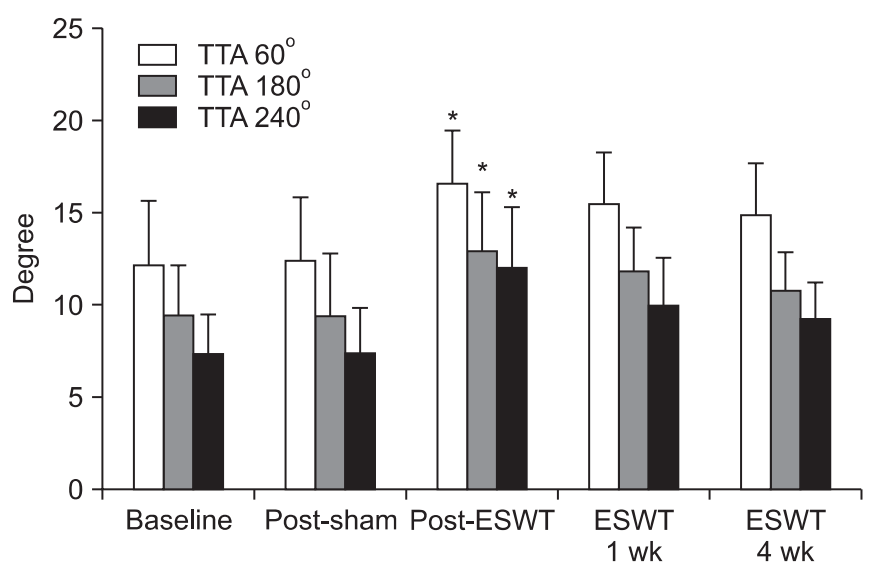

Fig. 5. Torque threshold angle (TTA) of ankle plantar flexor before extracorporeal shock wave therapy (ESWT) and after sham stimulation, and at immediately 1 week and 4 weeks after ESWT. *Statistically significant compared with baseline $(\mathrm{p}<0.05)$.

effect decreased over time and became not statistically significant 4 weeks later, although the degree of spasticity was lower than that of the baseline.

In the clinical assessment, the MAS of the ankle plantar flexor muscle statistically significantly decreased immediately after the treatment until one week after the treatment. In the biomechanical assessment, the two parameters were evaluated at the angular speeds of $60^{\circ}, 180^{\circ}$, and $240^{\circ}$ per second. Of these six evaluation items, four showed a statistically significant relief of spasticity only immediately after the treatment, whereas the remaining two showed a statistically significant relief of spasticity immediately after the treatment and one week after the treatment.

Among previous studies on upper limb spasticity, Manganotti and Amelio [8] stimulated the wrist flexor muscle once with 1,500 times and $0.030 \mathrm{~mJ} / \mathrm{mm}^{2}$, and the finger flexor muscle once with 800 times and $0.030 \mathrm{~mJ} / \mathrm{mm}^{2}$; the effects immediately after the treatment were maintained until 12 weeks after the treatment. Yoo et al. [11] stimulated the elbow flexor muscle and the wrist pronator muscle three times with $4 \mathrm{~Hz}, 1,000$ times, and 0.069 $\mathrm{mJ} / \mathrm{mm}^{2}$. The effects immediately after the treatment were maintained until four weeks after the treatment, differently from the results of the present study. Bae et al. [10] stimulated the belly and musculotendinous junction of the elbow flexor muscle three times with $4 \mathrm{~Hz}$, 1,200 times, and $0.12 \mathrm{~mJ} / \mathrm{mm}^{2}$; the spasticity was relieved statistically significantly only immediately after the treat- 
ment, but was not statistically significantly relieved one week and four weeks after the treatment, similar to the results of the present study. The reason for the differences in the results from the two studies mentioned earlier was assumed to be the differences in the mechanisms of shock wave generation, energy per unit area, the number of application, applied site and patients' durations of illness [10].

The mechanism of how ESWT relieves spasticity has not yet been clearly determined. Previous studies explained the mechanism as the effect of the generation of nitric oxides [14], the effect on spinal cord excitability [15], the effect of mechanical vibration [15], the effect on the golgi tendon organ [10], or the passive stiffness of muscles determined by inactive connective tissues [8]. Considering the known fact that the nitric oxides generated by ESWT involve the formation of neuromuscular junctions in the peripheral nervous system and play important roles in neurotransmission, memories, and synaptic plasticity in the central nervous system $[16,17]$ along with a recent report from an interesting study conducted with rats indicating that spasticity was relived because neuromuscular transmission was hindered by ESWT in neuromuscular junctions [18], nitric oxides seem to play core roles in spasticity-relieving mechanisms. Because F-wave and $\mathrm{H}$-reflex related variables showed no differences between before and after treatment in previous studies in which the neurophysiological evaluations were conducted, the effect on spinal cord excitability appears to be small $[8,9]$. Further, the hypothesis which states that shock waves would directly act on the golgi tendon organ to suppress motor nerve excitability [10] is also not quite likely. Due to the fact that the stimulation effects of mechanical vibration are temporary and maintained for short periods of time amounting approximately several hours, the stimulation effects of mechanical vibration should not be a major mechanism of the previous studies in which treatment effects were maintained for four weeks [11] or 12 weeks [8], as well as in the results of the present study that showed statistically significant relief of spasticity until one week after treatment. The elements which cause spasticity include stretch reflex and intrinsic stiffness of muscles [19]. The level of contribution of stretch reflex increases until 1-3 months after the onset of the disease in patients and decreases thereafter, whereas the level of contribution of intrinsic stiffness of muscles increases
$[20,21]$. The duration of treatment effect maintenance was different among previous studies. The mean periods after the onset of the disease of the subject patients were $9.5 \pm 6.8$ months in the study conducted by Yoo et al. [11], in which the treatment effects were maintained until four weeks after treatment. In the study conducted by Bae et al. [10], the mean periods were 22.0 \pm 8.2 months, in which the treatment effects were statistically significant only immediately after treatment. The reason for the differences in the duration of treatment effects is probably due to the fact that the treatment effects of ESWT were not maintained because the level of contribution of intrinsic stiffness of muscles was high when the period from the onset is long [10]. If the duration of illness is long, the stiffness of soft tissues around the joints and the joints themselves will become permanent and thus, repeated treatments for long periods of time will be necessary in order to change the intrinsic stiffness. In the present study, the mean period after the onset of the disease of the subject patients was $80.5 \pm 46.5$ days, which was shorter than that in previous studies. Spasticity was significantly relieved immediately after treatment. However, the treatment effects reduced with time and were not statistically significant at four weeks after treatment.

One of the reasons as to why the treatment effects were not maintained despite the fact that the duration of illness was relatively short should be associated with the differences in energy per unit area. The amount of energy per unit area that as delivered to the muscle was smaller in the present study compared to previous studies, because ESWT was applied to the gastrocnemius muscle, which has a relatively larger volume than the biceps brachii muscle. This is considered to be one of the reasons as to why the treatment effects were not maintained. Meanwhile, there were two studies conducted with cerebral palsy patients. Amelio and Manganotti [22] reported that when the soleus muscle and the medial and lateral gastrocnemius muscles were stimulated once with 1,500 times and $0.030 \mathrm{~mJ} / \mathrm{mm}^{2}$ in 12 child patients aged eight years on average, the treatment effects immediately after the treatment were maintained up to four weeks after the treatment, but were not statistically significant at three months after the treatment. In a study conducted by Vidal et al. [23], when the upper/lower limb muscles with severe spasticity were stimulated three times with 2,000 times and $0.10 \mathrm{~mJ} / \mathrm{mm}^{2}$ in 15 cerebral palsy patients aged 
31 years on average, the treatment effects immediately after the treatment were maintained up to two months after the treatment; however, the level of spasticity returned to that before the treatment at three months after the treatment. The reason as to why the treatment effects were maintained longer in the aforementioned two studies compared to the present study (although these studies were similar to the present study in that the treatment effects immediately after the treatment gradually decreased to become not statistically significant at three months after treatment) is considered to be the differences in energy per unit area. Because the average age of the subject patients was eight years, the volume of muscles in the subjects was smaller compared to adults in the case of the former study. In addition, the muscles were stimulated with a larger number of times and with higher energy than the present study, in the case of the latter study. Based on the foregoing, increasing the intensity of stimulation energy or conducting ESWT again within four weeks after treatment might be helpful in maintaining the treatment effects of ESWT on spasticity.

The present study is the first study that conducted a biomechanical assessment in order to determine the effects of ESWT on spasticity. Objective and quantitative spasticity assessments are essential for the evaluation of functional loss or treatment results as well as for the estimation of prognoses. There are three spasticity evaluation methods known thus far: clinical assessment, neurophysiological assessment, and biomechanical assessment [24]. The clinical assessment, which is frequently used in spasticity studies, are disadvantageous in that the subjectivity of the testers is quite likely to be involved in the evaluation, the reliability of the results is low, and the differences between grades are large and therefore, changes in spasticity cannot be sensitively represented [25]. The biomechanical assessment was introduced to overcome the limitations of the clinical assessment and to evaluate the spasticity both objectively and quantitatively. PET and TTA are useful parameters for this method [13]. This study also quantitatively evaluated the relief of spasticity after ESWT treatment by using the two parameters. The two parameters were evaluated at angular speeds of $60^{\circ} \%$ sec, $180^{\circ} / \mathrm{sec}$, and $240^{\circ} / \mathrm{sec}$.

In the case of PET at the angular speed $60^{\circ} / \mathrm{sec}$, compared to the basal evaluation, the values statistically significantly decreased only immediately after the treat- ment. At the angular speeds $180^{\circ} / \mathrm{sec}$ and $240^{\circ} / \mathrm{sec}$, compared to the basal evaluation, the values statistically significantly decreased immediately after the treatment and one week after the treatment. In the case of TTA at the angular speeds $60^{\circ} / \mathrm{sec}, 180^{\circ} / \mathrm{sec}$, and $240^{\circ} / \mathrm{sec}$, compared to the basal evaluation, the values statistically significantly increased only immediately after the treatment. PET is known to primarily reflect the intrinsic stiffness at low angular speeds as well as the entire spasticity, including both intrinsic stiffness and stretch reflex at high angular speeds. TTAs tend to primarily reflect the stretch reflex element of spasticity rather than the stiffness of joints themselves $[26,27]$. The TTA statistically significantly increased only immediately after the treatment at all angular speeds probably because the ESWT affects the intrinsic stiffness element more than the stretch reflex element of spasticity.

In the present study, ESWT was conducted in patients with lower limb spasticity after a stroke; not only were the effects immediately after the treatment checked but the maintenance of effects were also checked as well. In a previous study, the gastrocnemius muscle was stimulated once with 1,500 times and $0.1 \mathrm{~mJ} / \mathrm{mm}^{2}$ in 10 stroke patients; clinical assessment and neurophysiological assessment were conducted only immediately after the treatment [9]. Although the spasticity was statistically significantly relieved immediately after the treatment, whether the treatment effects were maintained thereafter was not checked. In the present study, the musculotendinous junction of the medial and lateral gastrocnemius were stimulated once a week for a total of three weeks at $4 \mathrm{~Hz}, 1,500$ times, and $0.089 \mathrm{~mJ} / \mathrm{mm}^{2}$ and the spasticity significantly relieved immediately after the treatment; however, the treatment effects decreased with time and became not statistically significant at four weeks after the treatment.

The present study has several limitations. First, the number of subject patients was insufficient. Second, the accurate acting mechanism of extracorporeal shock waves on spasticity was not determined. Third, only one treatment protocol was applied. To establish the appropriate treatment protocols in relation to the number of sessions, intensity, and application locations of treatment, additional studies will be necessary in order to apply diverse protocols to many patient groups and compare the effects. Given the fact that the duration of treat- 
ment effects varied with the length of time after the onset of stroke in patients, studies in which dividing patient groups based on the duration of illness and comparing the treatment effects should also be meaningful. Despite these several limitations, the fact that ESWT can be a useful alternative in spasticity treatment because it is noninvasive and involves much fewer adverse effects compared to the existing treatment methods and is effective in not only upper limb spasticity, as previously known before, but also in lower limb spasticity could be identified in the present study.

According to the clinical and biomechanical assessments, lower limb spasticity after stroke was statistically significantly relieved immediately after ESWT. Lower limb spasticity in subacute stroke patients was significantly improved immediately after ESWT. Although the therapeutic effect of ESWT reduced with time and therefore was not significant at four weeks after ESWT, the degree of spasticity was lower than that of the baseline. In the future, studies on treatment protocols which can optimize the effects of ESWT on lower limb spasticity should be conducted with a large number of patients.

\section{CONFLICT OF INTEREST}

No potential conflict of interest relevant to this article was reported.

\section{REFERENCES}

1. Lance JW. What is spasticity? Lancet 1990;335:606.

2. Mori F, Koch G, Foti C, Bernardi G, Centonze D. The use of repetitive transcranial magnetic stimulation (rTMS) for the treatment of spasticity. Prog Brain Res 2009;175:429-39.

3. Stevenson VL. Rehabilitation in practice: spasticity management. Clin Rehabil 2010;24:293-304.

4. Valchanou VD, Michailov P. High energy shock waves in the treatment of delayed and nonunion of fractures. Int Orthop 1991;15:181-4.

5. Wang CJ, Yang KD, Wang FS, Chen HH, Wang JW. Shock wave therapy for calcific tendinitis of the shoulder: a prospective clinical study with two-year followup. Am J Sports Med 2003;31:425-30.

6. Rompe JD, Theis C, Maffulli N. Shock wave treatment for tennis elbow. Orthopade 2005;34:567-70.
7. Rompe JD. Shock-wave therapy for plantar fasciitis. J Bone Joint Surg Am 2005;87:681-2.

8. Manganotti P, Amelio E. Long-term effect of shock wave therapy on upper limb hypertonia in patients affected by stroke. Stroke 2005;36:1967-71.

9. Sohn MK, Cho KH, Kim YJ, Hwang SL. Spasticity and electrophysiologic changes after extracorporeal shock wave therapy on gastrocnemius. Ann Rehabil Med 2011;35:599-604.

10. Bae H, Lee JM, Lee KH. The effects of extracorporeal shock wave therapy on spasticity in chronic stroke patients. J Korean Acad Rehabil Med 2010;34:663-9.

11. Yoo SD, Kim HS, Jung PK. The effect of shock wave therapy on upper limb spasticityin the patients with stroke. J Korean Acad Rehabil Med 2008;32:406-10.

12. Duncan PW, Propst M, Nelson SG. Reliability of the Fugl-Meyer assessment of sensorimotor recovery following cerebrovascular accident. Phys Ther 1983;63:1606-10.

13. Lee SJ, Han TR. A quantitative assessment of spasticity in hemiplegic patients using isokinetic dynamometer. J Korean Acad Rehabil Med 1998;22:784-92.

14. Mariotto S, Cavalieri E, Amelio E, Ciampa AR, de Prati AC, Marlinghaus E, et al. Extracorporeal shock waves: from lithotripsy to anti-inflammatory action by NO production. Nitric Oxide 2005;12:89-96.

15. Leone JA, Kukulka CG. Effects of tendon pressure on alpha motoneuron excitability in patients with stroke. Phys Ther 1988;68:475-80.

16. Mariotto S, de Prati AC, Cavalieri E, Amelio E, Marlinghaus E, Suzuki H. Extracorporeal shock wave therapy in inflammatory diseases: molecular mechanism that triggers anti-inflammatory action. Curr Med Chem 2009;16:2366-72.

17. Mariotto S, Menegazzi M, Suzuki H. Biochemical aspects of nitric oxide. Curr Pharm Des 2004;10:1627-45.

18. Kenmoku T, Ochiai N, Ohtori S, Saisu T, Sasho T, Nakagawa $\mathrm{K}$, et al. Degeneration and recovery of the neuromuscular junction after application of extracorporeal shock wave therapy. J Orthop Res 2012;30:1660-5.

19. Mirbagheri MM, Barbeau H, Ladouceur M, Kearney RE. Intrinsic and reflex stiffness in normal and spastic, spinal cord injured subjects. Exp Brain Res 2001;141:446-59.

20. Fellows SJ, Ross HF, Thilmann AF. The limitations of the tendon jerk as a marker of pathological stretch 
reflex activity in human spasticity. J Neurol Neurosurg Psychiatry 1993;56:531-7.

21. Given JD, Dewald JP, Rymer WZ. Joint dependent passive stiffness in paretic and contralateral limbs of spastic patients with hemiparetic stroke. J Neurol Neurosurg Psychiatry 1995;59:271-9.

22. Amelio E, Manganotti P. Effect of shock wave stimulation on hypertonic plantar flexor muscles in patients with cerebral palsy: a placebo-controlled study. J Rehabil Med 2010;42:339-43.

23. Vidal X, Morral A, Costa L, Tur M. Radial extracorporeal shock wave therapy (rESWT) in the treatment of spasticity in cerebral palsy: a randomized, placebo-controlled clinical trial. NeuroRehabilitation
2011;29:413-9.

24. Kim DY, Park CI, Na SI, Park YS. Quantitative assessment of the effect of tizanidine on spasticity in stroke patients. J Korean Acad Rehabil Med 2003;27:471-9.

25. Blackburn M, van Vliet P, Mockett SP. Reliability of measurements obtained with the Modified Ashworth Scale in the lower extremities of people with stroke. Phys Ther 2002;82:25-34.

26. Odeen I, Knutsson E. Evaluation of the effects of muscle stretch and weight load in patients with spastic paraplegia. Scand J Rehabil Med 1981;13:117-21.

27. Lee SJ, Kwon BS, Park CH. The effect of passive stretching on the spasticity of ankle plantar flexor muscles. J Korean Acad Rehabil Med 2001;25:987-92. 